\title{
Determination of left ventricular volumes and ejection fraction by nuclear mognetic resonance
}

\author{
imaging
}

To determine the ability of nuclear magnetic resonance (NMR) Imaging to aseess left ventricular (LV) volumes and ejection traction (EF), we studied 24 patients within 48 hours of single-plane LV angiography. In all patients, a transverse, single-plane MMR acquilation technique was employed with LV end-diastolic (ED) and end-systolic (ES) volumes (V) calctiated by a modified area-length algorithm. In nine patients, a multidice acquisition technique was employed with LVEDV and LVESV calculated by a SImpson's rule algorithm. NMR-determined LVV and EF correlated reasonably well with anglographic values (LVEDV: $r=0.75$; LVESV; $r=0.90$; and LVEF: $r=0.76$ ). The single-plane NMP technique significantly underestimated LVEDV $(p<0.01)$, whereas no significant difference was demonstrated for LVESV. As a result, anglographic LVEF was significantly underestimated $(p<0.05)$. This underestimation is thely related to off-axis imaging and to the geometric constraints of a single-plane algorithm. In comparing multillice Nin to angiographic data, no significant difference was demonstrated for LVEDV, LVESV, or LVEF. Thus, quantitation of LVV and EF with NMA is feasible, and comparison to anglographic volumes is simllar to results reported from other noninvasive imaging modaltiles. Improvement in current acquisition techniques and software should result in further quantitative potentlal. (AM HEART J 1987;113:24.)

Lee R. Dilworth, M.D., Alex M. Aisen, M.D., G. B. John Mancini, M.D., F.R.C.P.(C), Ian Lande, M.D., and Andrew J. Buda, M.D. Ann Arbor, Mich.

The assessment of left ventricular (LV) function is fundamental to the diagnostic evaluation, prognostication, and therapy of patients with cardiac disease. Traditionally, LV volume and ejection fraction have been important parameters in this assessment. Ever since Sandler, Dodge, et al. ${ }^{1,2}$ validated angiographic methods of measuring $\mathrm{LV}$ volumes and ejection fraction, a number of noninvasive techniques have been used to estimate these parameters. Both echocardiographic and gated blood pool estimates of $\mathrm{LV}$ volume have yielded variable results when compared to angiographic measurements. Nuclear magnetic resonance (NMR) imaging is a new technique that has been used primarily for tissue characterization and qualitative structural analyses. ${ }^{3}$ However, gated

From the Cardiology Division, Department of Internal Medicine and Department of Radiology, University of Michigan Medical School.

This work was supported in part by a Grant-in-Aid from the American Heart Association of Michigan; by Grant HL29716 from the National Heart, Lung, and Blood Institute, National Institutes of Health, Bethesda, Md.; and by The Veterans Administration, Washington, D.C.

Received for publication Jan. 30, 1986; accepted May 26, 1986.

Reprint requests: Andrew J. Buda, M.D., University of Michigan Medical Center, Cardiology Division, 1500 E. Medical Center Dr., TC-3910, Ann Arbor, MI 48019-0366. cardiac NMR images provide excellent endocardial definition and thus should be potentially useful for quantitating $L V$ volumes and ejection fraction. To determine the accuracy of NMR determination of LV volumes and ejection fraction, we used two methods for determining these parameters by NMR and compared them to results from standard methods that used LV angiography.

\section{METHODS}

Patient population. Twenty-seven patients (17 men and 10 women, mean age 59 years, range 36 to 70 years) in normal sinus rhythm were studied prospectively. After informed consent had been obtained, all patients underwent NMR and routine cardiac catheterization, including conventional or digital angiography, within 48 hours. Three patients, two with technically unsatisfactory NMR studies (due to an irregular cardiac rhythm resulting in poor image quality) and one with a technically inadequate ventriculogram (due to post injection ventricular tachycardia), were excluded from further analysis. Among the remaining 24 patients, six had normal cardiac evaluations, 14 had coronary artery disease (five with a history of myocardial infarction), two had variant angina, one had dilated cardiomyopathy, and one had idiopathic hypertrophic subaortic stenosis.

MMR acquialtion techniques and quantitative analysis. 

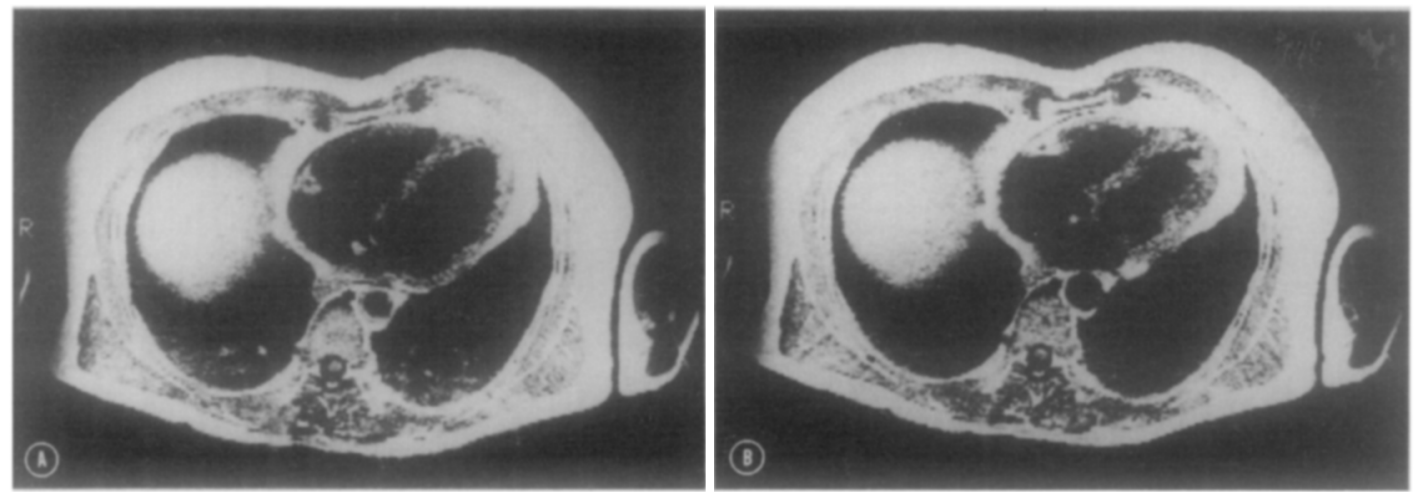

Fig. 1. Transverse nuclear magnetic resonance images (acquired by the single-plane technique) at the midventricular level gated to end diastole $(A)$ and end systole $(B)$. This patient had coronary artery disease, but normal left ventricular function by cardiac catheterization.

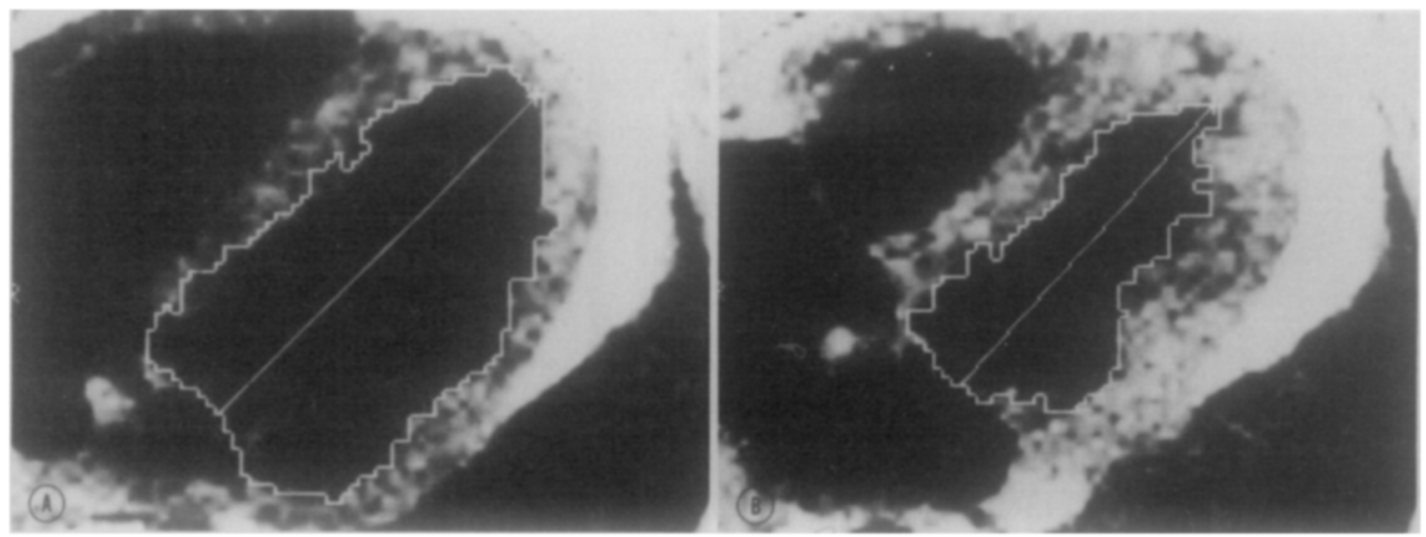

Fig. 2. Transverse nuclear magnetic resonance images (same as Fig. 1), after magnification and tracing of left ventricular endocardial surface and long axis, gated to end diastole $(A)$ and end systole $(B)$. Trabeculations and papillary muscles were excluded from planimetered areas. The long axis was measured from the midpoint of the mitral valve plane to the left ventricular "apex."

Imaging was performed with a 0.35 tesla superconducting magnet (Diasonics MT/S, Diasonics, Milpitas, Calif.). Nonferromagnetic ECG leads were placed on the right and left subclavian and right abdominal regions. Gated, spinecho NMR was performed with an echo delay (TE) of 28 msec between the application of the initial radiofrequency pulse and the receipt of the corresponding signal (spinecho). The pulse sequence repetition time (TR) was determined by each subject's heart rate (60 to $100 \mathrm{bpm}$ ) and thus varied from about 0.6 to 1.0 second. Two NMR acquisition techniques (transverse single-plane and transverse multislice) were employed. A single-plane study required a total of 30 to 40 minutes and each multislice study required 50 to 60 minutes. The transverse singleplane acquisition technique was used in all 24 patients. Initially, a midventricular slice was chosen from five ungated images acquired at adjacent levels of the $\mathrm{LV}, 1 \mathrm{~cm}$ apart. Next, a gated end-diastolic image (10 msec after the $R$ wave) was acquired at this level (Fig. 1, A). Gating was then reset for end systole (estimated by the midpoint of the downslope of the $T$ wave ${ }^{4}$ ), again with acquisition at the midventricular level (Fig. 1, B).

Quantitative analysis of these first spin-echo images was performed with a planimetry program. After magnification, the endocardial border of the LV was traced, excluding the papillary muscles, and the LV area was computed. The long axis was measured from the midpoint of the mitral valve plane to the LV "apex" (Fig. $2, A$ and $B)$. LV volumes were calculated by means of a modified area-length algorithm ${ }^{5}$ : $\quad V=8 / 3 \pi \times A^{2} / L \quad(1)$, where $\mathrm{V}=\mathrm{LV}$ volume, $\mathrm{A}=$ area of transverse plane (through the center of the $\mathrm{LV}$ ), and $\mathrm{L}=$ long axis (of same transverse plane).

The multislice acquisition technique was employed in 9 of the 24 patients. Initially, five images (at consecutive 100 msec intervals) were acquired at each of 10 levels (Fig. 3). The end-diastolic (the first frame) and the approximate end-systolic (the frame with the smallest $\mathrm{LV}$ area) images were chosen at all levels through the left ventricle and were planimetered for area, with volumes calculated by a 


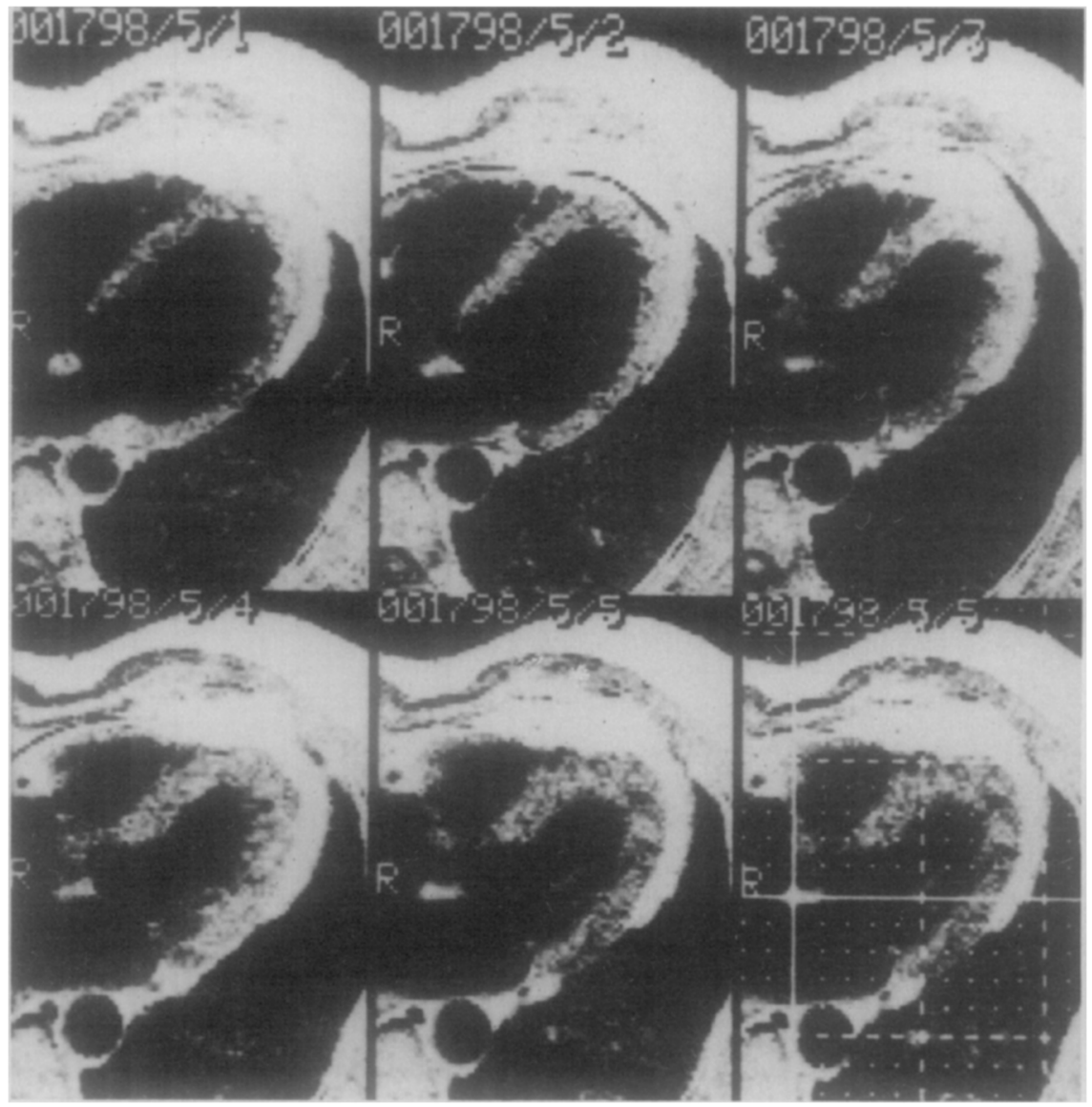

Fig. 3. Transverse nuclear magnetic resonance images (acquired by the multislice technique) at the midventricular level. The first image was gated $10 \mathrm{msec}$ after the $R$ wave. The next four images were acquired at the same level at consecutive $100 \mathrm{msec}$ intervals. The final image is identical to the fifth except with a grid overlaid. The end-diastolic (first frame) and approximate end-systolic (fourth frame, which has the smallest left ventricular area) images were analyzed as shown in Fig. 2. This patient had normal cardiac catheterization.

Simpson's rule algorithm: $V=\left(A_{1}+A_{2}+\ldots+A_{n-1}\right) h+$ $(A n) h / 2+(\pi / 6) h^{3}(2)$ where $V=L V$ volume, $A_{1}=$ planimetered area of $L V$ at first level (base of $L V$ ), $A n=$ planimetered area of $L V$ at level " $n$ " ( $L V$ apex), and $h=$ distance between the centers of two adjacent slices $(1 \mathrm{~cm})$.

The interobserver variability of the transverse, singleplane technique was assessed in 10 of 24 studies (chosen at random) by two investigators who interpreted the same study and measured the volumes independently of each other. Intraobserver variability was evaluated by one of the investigators repeating the measurements in 10 of 24 studies selected at random at least 1 month after the first interpretation and without knowledge of the first measurements.

Anglographic acquisition techniques and quantitative analyels. Single-plane LV angiograms were acquired in all 24 patients (by conventional or digital technique) in the 30-degree right anterior oblique (RAO) projection at 60 frames/sec. For conventional contrast angiography, 35 to $50 \mathrm{ml}$ of Renografin-76 was injected at a flow rate of 10 to $12 \mathrm{ml} / \mathrm{sec}$. For digital angiography, Renografin was mixed with normal saline $1: 1$, and 35 to $50 \mathrm{ml}$ was injected at the same rate. The $L V$ silhouette was traced at end diastole (largest cavity size) and at end-systole (smallest cavity size). Only beats in sinus rhythm not preceded by a premature contraction were used for analysis. LV volumes were calculated by the modified area-length algorithm, ${ }^{5}$ and the following RAO ventriculogram correction factor ${ }^{7}$ was applied: $\mathrm{V}=0.81 \times \mathrm{V}^{\prime}+1.9 \mathrm{ml}$ (3) where $\mathrm{V}=$ corrected $L V$ volume and $V^{\prime}=$ uncorrected $L V$ volume (as calculated from the modified area-length algorithm).

Statlotical analyals. All data are expressed as mean \pm standard deviation. Statistical correlations between data were made with linear regression analysis. Student's paired $t$ test was employed to determine statistical significance in comparing the NMR to angiographic data. Results were considered significant if $p$ was less than 0.05 .

\section{RESULTS}

The angiographic and NMR data are listed for individual patients in Tables I and II. Patients No. 1 
Table I. Volume and ejection fraction data

\begin{tabular}{|c|c|c|c|c|c|c|c|}
\hline \multirow{2}{*}{$\begin{array}{c}\text { Patient } \\
\text { no. }\end{array}$} & \multirow[b]{2}{*}{ Diagnosis } & \multicolumn{2}{|c|}{$L V E D V(m l)$} & \multicolumn{2}{|c|}{$L V E S V(m l)$} & \multicolumn{2}{|c|}{$L V E F(\%)$} \\
\hline & & Angio & $N M R$ & Angio & $N M R$ & Angio & $N M R$ \\
\hline 1 & IHSS & 51 & 48 & 8 & 14 & 84 & 71 \\
\hline 2 & $\mathrm{CAD},-\mathrm{MI}$ & 155 & 105 & 29 & 22 & 81 & 79 \\
\hline 3 & $\mathrm{CAD},+\mathrm{MI}$ & 122 & 143 & 55 & 82 & 55 & 43 \\
\hline 4 & $\mathrm{CAD},+\mathrm{MI}$ & 101 & 88 & 46 & 33 & 54 & 63 \\
\hline 5 & $\mathrm{CAD},-\mathrm{MI}$ & 153 & 153 & 63 & 49 & 59 & 68 \\
\hline 6 & $\mathrm{CAD},+\mathrm{MI}$ & 156 & 105 & 33 & 26 & 79 & 75 \\
\hline 7 & $\mathrm{CAD},-\mathrm{MI}$ & 144 & 147 & 74 & 59 & 49 & 60 \\
\hline 8 & VA & 170 & 146 & 72 & 57 & 58 & 61 \\
\hline 9 & $\mathrm{CAD},-\mathrm{MI}$ & 110 & 122 & 37 & 37 & 66 & 70 \\
\hline 10 & $\mathrm{NL}$ & 183 & 114 & 53 & 49 & 71 & 57 \\
\hline 11 & NL & 123 & 99 & 32 & 38 & 74 & 62 \\
\hline 12 & $\mathrm{CAD},-\mathrm{MI}$ & 95 & 81 & 19 & 27 & 80 & 67 \\
\hline 13 & $\mathrm{CAD},+\mathrm{MI}$ & 174 & 180 & 61 & 71 & 65 & 61 \\
\hline 14 & $\mathrm{CAD},-\mathrm{MI}$ & 142 & 128 & 49 & 36 & 65 & 72 \\
\hline 15 & $\mathrm{CAD},-\mathrm{MI}$ & 146 & 89 & 36 & 29 & 75 & 67 \\
\hline 16 & NL & 82 & 92 & 26 & 22 & 68 & 76 \\
\hline 17 & NL & 144 & 168 & 38 & 38 & 74 & 77 \\
\hline 18 & VA & 171 & 134 & 59 & 51 & 65 & 62 \\
\hline 19 & NL & 128 & 115 & 38 & 34 & 70 & 70 \\
\hline 20 & NL & 93 & 83 & 21 & 27 & 77 & 67 \\
\hline 21 & CAD,-MI & 118 & 134 & 29 & 30 & 75 & 78 \\
\hline 22 & $\mathrm{CAD},-\mathrm{MI}$ & 98 & 73 & 33 & 33 & 66 & 55 \\
\hline 23 & $\mathrm{CAD},+\mathrm{MI}$ & 165 & 160 & 49 & 75 & 70 & 53 \\
\hline 24 & $\mathrm{DCM}$ & $\underline{199}$ & $\underline{202}$ & $\underline{145}$ & 180 & $\underline{27}$ & $\underline{11}$ \\
\hline Mean \pm S.D. & & $134 \pm 36$ & $121 \pm 37 \dagger$ & $46 \pm 27$ & $47 \pm 33$ & $67 \pm 12$ & $64 \pm 14^{*}$ \\
\hline
\end{tabular}

Abbreviations: $L V E D V=$ left ventricular end-diastolic volume; LVESV $=$ left ventricular end-systolic volume; LVEF $=$ left ventricular ejection fraction; IHSS = idiopathic hypertrophic subaortic stenosis; $\mathrm{CAD}=$ coronary artery disease; $-\mathrm{MI}=$ no history of myocardial infarction; $+\mathrm{MI}=\mathrm{history}$ of myocardial infarction; VA = variant angina; $\mathrm{DCM}=$ dilated cardiomyopathy; $\mathrm{NL}=$ normal cardiac evaluation; Angio $=$ angiography; $\mathrm{NMR}=$ nuclear magnetic resonance imaging.

With the use of single-plane NMR, ${ }^{*} p<0.05,+p<0.01$.

Table II. Volume and ejection fraction data

\begin{tabular}{|c|c|c|c|c|c|c|c|}
\hline \multirow{2}{*}{$\begin{array}{c}\text { Patient } \\
\text { no. }\end{array}$} & \multirow[b]{2}{*}{ Diagnosis } & \multicolumn{2}{|c|}{$L V E D V(m l)$} & \multicolumn{2}{|c|}{$L V E S V(m l)$} & \multicolumn{2}{|c|}{ LVEF (\%) } \\
\hline & & Angio & $N M R^{*}$ & Angio & $N M R^{*}$ & Angio & $N M R^{*}$ \\
\hline 1 & IHSS & 51 & 66 & 8 & 19 & 84 & 71 \\
\hline 2 & $\mathrm{CAD},-\mathrm{MI}$ & 155 & 124 & 29 & 29 & 81 & 77 \\
\hline 3 & $\mathrm{CAD},+\mathrm{MI}$ & 122 & 167 & 55 & 58 & 55 & 65 \\
\hline 4 & $\mathrm{CAD},+\mathrm{MI}$ & 101 & 91 & 46 & 41 & 54 & 55 \\
\hline 5 & $\mathrm{CAD},-\mathrm{MI}$ & 153 & 142 & 63 & 62 & 59 & 56 \\
\hline 6 & $\mathrm{CAD},+\mathrm{MI}$ & 156 & 127 & 33 & 38 & 79 & 70 \\
\hline 7 & $\mathrm{CAD},-\mathrm{MI}$ & 144 & 105 & 74 & 59 & 49 & 44 \\
\hline 8 & VA & 170 & 163 & 72 & 45 & 58 & 72 \\
\hline 9 & $\mathrm{CAD},-\mathrm{MI}$ & $\underline{110}$ & $\underline{114}$ & $\underline{37}$ & $\underline{38}$ & $\underline{66}$ & $\underline{67}$ \\
\hline Mean \pm S.D. & & $129 \pm 37$ & $122 \pm 33$ & $46 \pm 22$ & $43 \pm 14$ & $65 \pm 13$ & $64 \pm 10$ \\
\hline
\end{tabular}

Abbreviations: $L V E D V$ = left ventricular end-diastolic volume; $L V E S V=$ left ventricular end-systolic volume; LVEF = left ventricular ejection fraction; IHSS = idiopathic hypertrophic subaortic stenosis; $\mathrm{CAD}=$ coronary artery disease; $-\mathrm{MI}=$ no history of myocardial infarction; $+\mathrm{MI}=$ history of myocardial infarction; VA = variant angina, $\mathrm{DCM}=$ dilated cardiomyopathy; $\mathrm{NL}=$ normal cardiac evaluation; Angio $=$ left ventricular angiography; NMR = nuclear magnetic resonance imaging.

*With the use of multislice NMR.

to 9 had both NMR acquisition techniques (singleplane and multislice) performed. The single-plane NMR technique significantly understimated angiographic left ventricular end-diastolic volume
(LVEDV) $(p<0.01)$. No significant difference was demonstrated between NMR single-plane and angiographic left ventricular end-systolic volume (LVESV). As a result of the NMR underestimation 
Table III. Linear regression analysis

\begin{tabular}{llrrr}
\hline Variable & \multicolumn{1}{c}{$x$ vs $y$} & No. & r & Equation \\
\hline LVEDV & Angio vs NMR (Both methods) & 33 & 0.75 & $\mathrm{y}=0.74 \times+22.7$ \\
LVEDV & Angio vs NMR (Single-plane) & 24 & 0.76 & $\mathrm{y}=0.79 \times+15.6$ \\
LVEDV & Angio vs NMR (Multislice) & 9 & 0.73 & $\mathrm{y}=0.64 \times+39.2$ \\
& & & \\
LVESV & Angio vs NMR (Both methods) & 33 & 0.90 & $\mathrm{y}=1.04 \times-2.2$ \\
LVESV & Angio vs NMR (Single-plane) & 24 & 0.92 & $\mathrm{y}=1.14 \times-6.1$ \\
LVESV & Angio vs NMR (Multislice) & 9 & 0.88 & $\mathrm{y}=0.58 \times+16.3$ \\
& & & \\
LVEF & Angio vs NMR (Both methods) & 33 & 0.76 & $\mathrm{y}=0.80 \times+10.3$ \\
LVEF & Angio vs NMR (Single-plane) & 24 & 0.78 & $\mathrm{y}=0.89 \times+4.0$ \\
LVEF & Angio vs NMR (Multislice) & 9 & 0.76 & $\mathrm{y}=0.60 \times+25.0$ \\
\hline
\end{tabular}

Abbreviations as in Tables I and II.

of LVEDV, angiographic left ventricular ejection fraction (LVEF) was significantly underestimated $(p<0.05)$ by the NMR single-plane technique. In comparing multislice NMR data to angiographic data, no significant difference was demonstrated for LVEDV, LVESV, or LVEF.

Table III summarizes the correlation coefficients and equations of the linear regression analysis for all data. A fair correlation was observed between LVEDV by NMR and LVEDV by angiography $(r=0.75, \mathrm{SEE} \pm 24 \mathrm{ml})$. The correlation with angiography was similar for both the NMR single-plane technique and the NMR multislice technique $(r=0.76, \mathrm{SEE} \pm 25 \mathrm{ml}$ vs $r=0.73, \mathrm{SEE} \pm 23 \mathrm{ml}$, respectively) (Fig. 4). A good correlation was observed between LVESV by NMR and LVESV by angiography $(r=0.90, \mathrm{SEE} \pm 13 \mathrm{ml})$. The correlation with angiography was similar for both the NMR single-plane technique and the NMR multislice technique $(r=0.92$, SEE $\pm 13 \mathrm{ml}$ vs $r=0.88$, SEE $\pm 7 \mathrm{ml}$, respectively) (Fig. 5). A fair correlation was observed between LVEF by NMR and LVEF by angiography $(r=0.76, \mathrm{SEE} \pm 8 \%)$. Again, the correlation with angiography was similar for both the NMR single-plane technique and the NMR multislice technique $(r=0.78, \mathrm{SEE} \pm 9 \%$ vs $r=0.76$, SEE $\pm 7 \%$, respectively) (Fig. 6).

In the nine patients who had both NMR acquisition techniques performed, a good correlation was observed between LV volumes by the NMR singleplane and NMR multislice techniques (LVEDV: $r=0.88, S E E \pm 21 \mathrm{ml}$; and LVESV: $r=0.90$, $\mathrm{SEE} \pm 11 \mathrm{ml}$ ). No significant difference between these two techniques was demonstrated for LVEDV or LVESV.

Results of intraobserver and interobserver variability are summarized in Table IV. For LVEDV, the mean intraobserver variability was $13 \mathrm{ml}(10 \%)$ and the mean interobserver variability was $20 \mathrm{ml}$ (15\%). For LVESV, the mean intraobserver variability was $6 \mathrm{ml}(14 \%)$ and the mean interobserver variability was $8 \mathrm{ml}(17 \%)$.

\section{DISCUSSION}

Our data demonstrate that NMR-determined LV volumes and ejection fraction are quantifiable and correlate reasonably well with angiographic volumes and ejection fraction (LVEDV: $r=0.75$, LVESV: $r=0.90$, and LVEF: $r=0.76$ ). The transverse, single-plane NMR technique underestimated LVEDV $(p<0.01)$, whereas no significant difference was demonstrated for LVESV. As a result, angiographic LVEF was underestimated $(p<0.05)$. This underestimation is likely related both to off-axis NMR imaging, resulting in foreshortening of the long axis, and to the geometric constraints of a single-plane algorithm. In the nine patients who had both NMR acquisition techniques (single-plane and multislice) performed, no significant difference was demonstrated between the two methods for LVEDV, LVESV, or LVEF. (Multislice acquisition was performed in only nine patients because this technique became available only near the end of our study.) Although it was hoped that multislice acquisition would account for partial volume effects, correlation with angiography was no better than with the singleplane technique for each of the three parameters. This unexpected result may be explained by a number of factors. First, the multislice technique required determining area and long axis at several levels (usually five), thus increasing the potential measurement error. Second, the estimation of end systole was less precise with the multislice technique, as images at each level were available only at $100 \mathrm{msec}$ intervals. Finally, since a small number of patients were imaged over a narrow range of vol- 


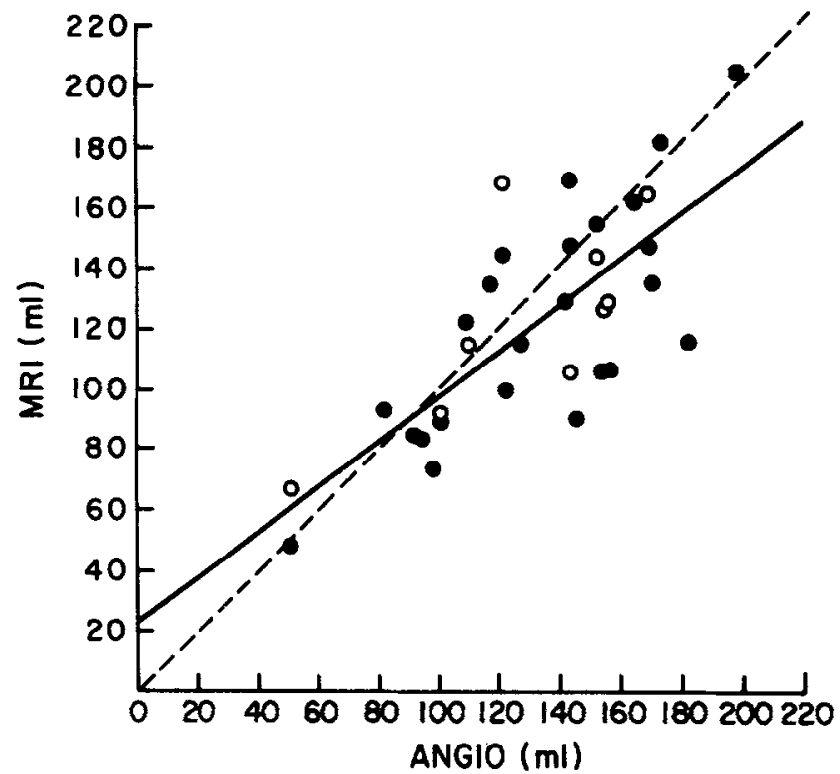

\begin{tabular}{lcccc} 
& $n$ & $r$ & $y$ & see \\
\cline { 2 - 5 } - Single-Plone & 24 & .76 & $0.79 x+15.6$ & 25 \\
- Multi-Slice & 9 & .73 & $0.64 x+39.2$ & 23 \\
$0+0$ & 33 & .75 & $0.74 x+22.7$ & 24
\end{tabular}

Fig. 4. Relationship between left ventricular end-diastolic volume as measured by angiography and by NMR. The line of identity is indicated by the dashed line. The plotted regression line includes data from both NMR acquisition techniques. $A N G I O=$ left ventricular angiography; $M R I=$ nuclear magnetic resonance imaging; $m l=$ milliliters.

umes, the correlation with angiographic values may have been limited on this basis. Despite these concerns, on-axis, multislice imaging in a larger group of patients may be expected to result in improved statistical correlation.

The results of this in vivo study of gated, cardiac NMR quantitation of $L V$ volumes are encouraging and complement the experience of others. A recent investigation $^{8}$ demonstrated that normal end-diastolic cardiac dimensions by NMR are reproducible and correlate with values obtained by two-dimensional echocardiography (2D echo). Preliminary results of two other studies demonstrated that NMR accurately measured volumes of latex casts of the human left ventricle, ${ }^{9}$ and also accurately measured $\mathrm{LV}$ function in seven patient studies with the use of a computerized three-dimensional reconstruction. ${ }^{10}$ Thus, increasing evidence supports the use of NMR to accurately quantitate $\mathrm{LV}$ dimensions, volumes, and function.

In recent years, radionuclide angiography and $2 \mathrm{D}$ echo have become the preferred noninvasive methods for $L V$ volume measurement. This study indi-

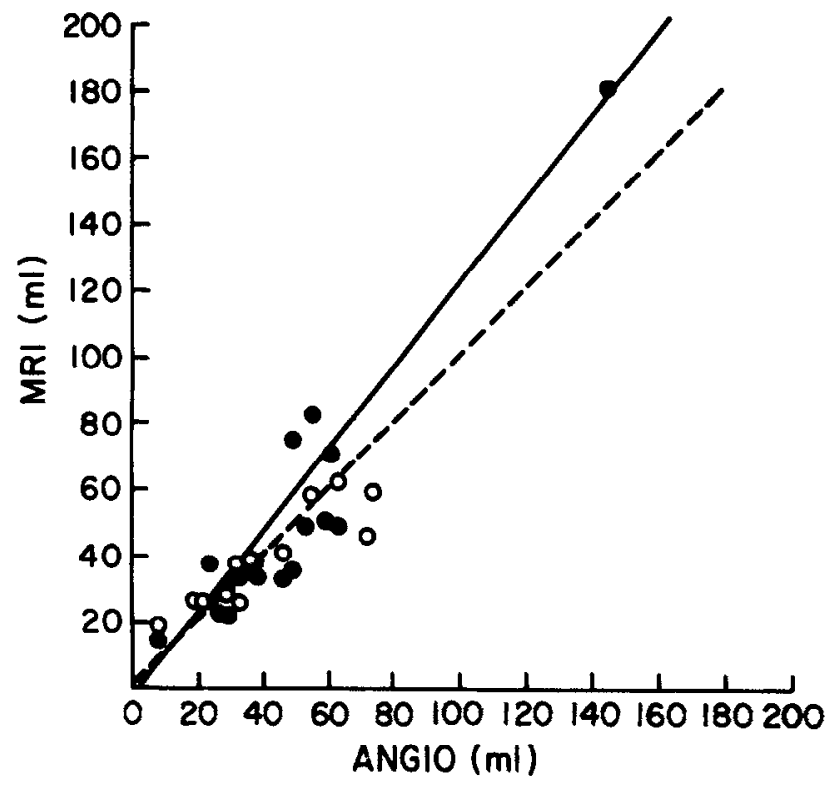

\begin{tabular}{lcccc} 
& $n$ & $r$ & $y$ & see \\
\cline { 2 - 5 } - Single-Plane & 24 & .92 & $1.14 x+6.1$ & 13 \\
- Multi-Slice & 9 & .88 & $0.58 x+16.3$ & 7 \\
-+0 & 33 & .90 & $1.04 x-2.2$ & 13
\end{tabular}

Fig. 5. Relationship between left ventricular end-systolic volume as measured by angiography and by NMR. The line of identity is indicated by the dashed line. The plotted regression line includes data from both NMR acquisition techniques. ANGIO = left ventricular angiography; $M R I=$ nuclear magnetic resonance imaging; $m l=$ milliliters.

cates that NMR determination of LV volumes is comparable to these other noninvasive techniques of volume assessment. Numerous studies ${ }^{11-15}$ have reported variable success in estimating angiographic $\mathrm{LV}$ volumes with $2 \mathrm{D}$ echo. Correlations have varied widely, depending upon the view(s) chosen for measurement of dimensions, the method of measuring the dimensions, the geometric algorithm used for volume calculation, the percentage of patients studied with cardiac disease, and perhaps most importantly, the quality of the images acquired. For example, Folland et al. ${ }^{13}$ observed a correlation coefficient of $r=0.61$ (SEE $\pm 52 \mathrm{ml}$ ) for LVEDV and $r=0.64$ (SEE $\pm 48 \mathrm{ml}$ ) for LVESV when comparing $2 \mathrm{D}$ echo volumes calculated by a single-plane ellipse algorithm to single-plane angiographic volumes. In contrast, Silverman et al., ${ }^{14}$ with the use of a biplane algorithm for both $2 \mathrm{D}$ echo and angiographic images in 20 children, reported excellent correlations (LVEDV: $r=0.97$, and LVESV: $r=0.91$ ) for volumes, but LVEF correlated less well $(r=0.82)$. Several 2D echo studies report underestimation of angiographic ventricular size, often by as 


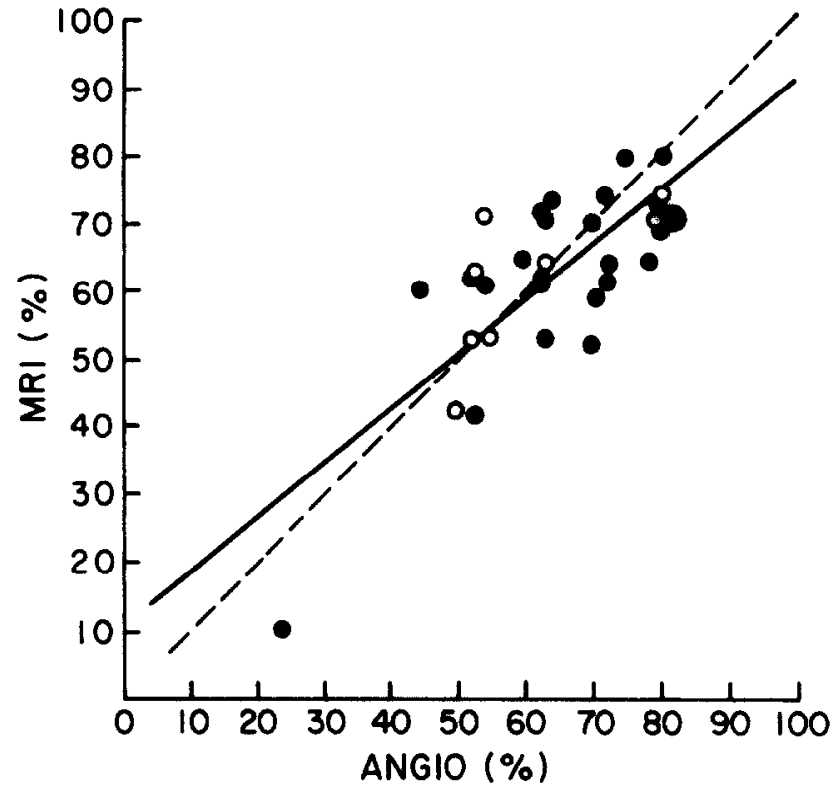

\begin{tabular}{lcccc} 
& $n$ & $r$ & $y$ & see \\
\cline { 2 - 5 } - Single-Plone & 24 & .78 & $0.89 x+4.0$ & 9 \\
- Multi-Slice & 9 & .76 & $0.60 x+25.0$ & 7 \\
- 0 & 33 & .76 & $0.80 x+10.3$ & 8
\end{tabular}

Fig. 6. Relationship between left ventricular ejection fraction as measured by angiography and by NMR. The line of identity is indicated by the dashed line. The plotted regression line includes data from both NMR acquisition techniques. $A N G I O=$ left ventricular angiography; $M R I=$ nuclear magnetic resonance imaging.

much as $30 \%,{ }^{15}$ in patients with normal or diseased hearts.

In addition, several radionuclide methods for measuring $\mathrm{LV}$ volume have been developed, but none has gained universal clinical acceptance. Although some investigators ${ }^{16,17}$ have reported good results with area-length algorithms on equilibriumgated cardiac blood pool images, such volume determinations are prone to error due to difficulty in identifying $L V$ boundaries and true length, amplifcation of inaccurate measurements because of miniaturized display sizes for most gated images, and the limitations inherent in shape assumption. Other authorg ${ }^{18-20}$ have reported excellent correlations $(r=0.95$ to 0.99 ) with contrast angiography when count-based ventricular volume determination by equilibrium blood pool scanning is employed. A major advantage of this technique is its independence from geometric assumptions regarding the $L V$ cavity. A more recent report from our institution, ${ }^{21}$ however, indicates that a sizable inherent error may be associated with individual count-based LV vol-
Table IV. Intraobserver and interobserver variability (single-plane NMR technique)

\begin{tabular}{cccc}
\hline & No. & $\mathrm{r}$ & Equation \\
\hline A. Intraobserver variability & & \\
LVEDV & 10 & 0.94 & $\mathrm{y}=0.82 \times+11.4$ \\
LVESV & 10 & 0.96 & $\mathrm{y}=0.90 \times+2.0$ \\
B. Interobserver variability & & \\
LVEDV & 10 & 0.86 & $\mathrm{y}=0.86 \times-13.7$ \\
L.VESV & 10 & 0.97 & $\mathrm{y}=1.19 \times-13.3$ \\
\hline
\end{tabular}

Abbreviations as in Tables I and II.

ume measurements. Thus, although a number of noninvasive techniques for $\mathrm{LV}$ volume determination have been validated, each method has unique problems in accurately defining true volume.

There are a number of limitations and potential sources of error in our study that may have affected our results. First, each NMR image was acquired in a transverse plane, since "on-axis" imaging was not yet possible at the time of our patient studies. The images analyzed, therefore, were neither true longor short-axis views. The area-length algorithm ${ }^{5}$ used for computation of volumes assumes a long-axis view through the LV apex, and thus LV area and "longaxis" measured in our NMR studies would be expected to underestimate the true cardiac dimensions and volume.

Second, the time delay between acquisition of NMR and angiographic images, though less than 48 hours for each patient, may have resulted in actual LV volume differences, due to changes in the individual's heart rate and hemodynamic state. Third, there are inherent differences between NMR and contrast angiography; NMR is a tomographic technique, whereas angiography is a projection technique. Thus, improper selection of a midventricular level from initial ungated NMR images may result in underestimation of area, and thus of volume. If the patient shifts position during or between individual NMR image acquisition periods (each lasting 6 to 10 minutes), inaccurate estimates may result. (We repeated imaging in the only case where this was a problem.) Furthermore, the NMR image is an "average picture" of numerous beats, whereas the angiographic image is a picture of a single beat.

Fourth, although NMR acquisition is gated, there are inherent difficulties with present acquisition techniques. As a result, the images acquired and chosen for analysis probably do not represent true end diastole and end systole. The first spin-echo signal is received $28 \mathrm{msec}$ after the preset gating interval. Thus for both single-plane and multislice techniques, with a gating interval of $10 \mathrm{msec}$ after 
the $R$ wave, end diastole is probably approximated well. For the single-plane technique, the end-systolic gating interval was set to coincide with the midpoint of the downslope of the $\mathrm{T}$ wave. ${ }^{4}$ However, for the multislice technique, the end-systolic image was chosen from five consecutive images acquired at 100 msec intervals, introducing a potential error due to the limited temporal resolution.

Fifth, there may be difficulties related to the definition of the endocardial borders of the left ventricle. A recent study ${ }^{22}$ reported obscuration of endocardial boundaries by high intraluminal flow signals in NMR images from patients with LV wall motion abnormalities, particularly with second spinecho imaging, presumably due to stasis of blood bordering of involved segment(s). In the vast majority of our images, we observed excellent endocardial definition without the problem of obscuration, probably due to first spin-echo imaging and also because only $25 \%$ of the patients had wall motion abnormalities. However, in some instances, arbitrary tracing of the mitral valve plane or short portions of the endocardial boundary was necessary due to poor definition, particularly in images acquired near the base or apex by the multislice technique.

Sixth, the assumption that angiographically determined volume represents a gold standard may not be reasonable. As a projection technique, angiography is subject to error from divergence of $x$-ray beams and from magnification changes. Although correction factors are employed to account for these problems, some error is introduced. Intraventricular contrast injection has been shown to produce hemodynamic changes that adversely affect $L V$ performance because of the contrast agent's negative inotropic and volume loading effects. ${ }^{23}$ We tried to minimize this problem by analyzing one of the first five beats after contrast injection. Also, the tracing of endocardial surfaces from angiographic images may be error-prone due to contrast invagination around trabeculae and papillary muscles.

Finally, as with most other imaging modalities including angiography, this method of NMR analysis relates measured $\mathrm{LV}$ dimensions to volume by assuming a geometric configuration of the left ventricle, as well as a single, fixed relationship between LV dimensions and volume at all parts of the cardiac cycle. A recent analysis ${ }^{24}$ disputes these basic assumptions, noting that the LV shape changes as cavity volume changes and that this assumption of a fixed relationship between dimensions and volume becomes even more tenuous when changes in volumes are measured at frequencies greater than the heart rate.
We emphasize that the NMR imaging techniques utilized in this study are crude and represent the initial capabilities of first-generation imaging systems. Data acquisition was time-consuming and results are without immediate clinical utility. Our data, however, do suggest the feasibility of $\mathrm{LV}$ volume assessment with this new modality. Ongoing improvements in NMR software and acquisition techniques should result in further improved accuracy of volume determinations. ${ }^{10}$ Methods for obtaining oblique on-axis images with NMR, either by reorienting the direction of the magnetic field gradients or by repositioning the patient, ${ }^{25,26}$ are now commercially available. The relatively long acquisition time for NMR can be shortened by utilizing echo-planar techniques. ${ }^{27}$ Currently, however, this method sacrifices resolution for a reduction in acquisition time. A combination of echo-planar techniques with the use of volume hybrid methods ${ }^{28}$ might shorten the time required for a study without significantly compromising image quality, even permitting creation of tomographic cine films at a chosen level. ${ }^{29}$

In summary, quantitation of $\mathrm{LV}$ volumes and ejection fraction with NMR is feasible. Statistical comparison with angiographic volumes is similar to initial results reported for other noninvasive imaging modalities. Although it is unlikely that NMR will replace currently used modalities at the present time, ongoing development of NMR acquisition techniques and software should improve the quantitation of LV volumes and global function, as well as regional analysis of $L V$ wall motion and thickening. The ability of proton NMR to accurately measure ventricular performance as well as delineate myocardial tissue characterization suggests that it should become an increasingly important cardiac imaging technique.

We thank Ms. Sheree Wilson for her expert assistance in typing the manuscript.

\section{REFERENCES}

1. Dodge HT, Sandler H, Ballew DW, Lord JD. The use of biplane angiocardiography for the measurement of left ventricular volume in man. AM HEART J 1960;60:762.

2. Sandler $H$, Dodge HT. Use of single plane angiocardiograms for the calculation of left ventricular volume in man. AM HEART J 1968;75:325.

3. Tscholakoff $\mathbf{D}$, Higgins $C B$. Magnetic resonance imaging of the heart. Int J Cardiac Imaging 1985;1:73.

4. Strauss HW, Zaret BL, Hurley PJ, Natarajan TK, Pitt B. A scintiphotographic method for measuring left ventricular ejection fraction in man without cardiac catheterization. Am J Cardiol 1971;28:575.

5. Grossman W, ed. Cardiac catheterization and angiography. 2nd ed. Philadelphia: Lea \& Febiger, Publishers, 1980; 237.

6. Wyatt HL, Heng MK, Meerbaum S, Hestenes JD, Cobo JM, Davidson RM, Corday E. Cross sectional echocardiography. I. 
Analysis of mathematical models for quantifying mass of the left ventricle in dogs. Circulation 1979;60:1104.

7. Kennedy JW, Trenholme SE, Kasser IS. Left ventricular volume and mass from single-plane cineangiocardiograms: A comparison of antero-posterior and right anterior oblique methods. AM HEART J 1970;80:343.

8. Byrd BF, Schiller NB, Botvinick EH, Higgins CB. Normal cardiac dimensions by magnetic resonance imaging. Am J Cardiol 1985;55:1440.

9. Rehr RB, Malloy CR, Peshock RM, Filipchuk N. Quantitation of left ventricular volumes by magnetic resonance imaging (abstr). Circulation 1984;70(suppl II):II-447.

10. Thompson R, Edelman R, Kantor H, Leavitt M, Miller D, Okada R, Brady T, Dinsmore R. Calculation of left ventricular volumes and ejection fraction from magnetic resonance tomograms using a computerized 3-D reconstruction: Comparison with ventriculography (abstr). J Am Coll Cardiol 1986;7:174A.

11. Wyatt HL, Heng MK, Meerbaum S, Gueret $P$, Hestenes J, Dula E, Corday E. Cross-sectional echocardiography. II. Analysis of mathematical models for quantifying volume of the formalin-fixed left ventricle. Circulation 1980;61:1119.

12. Kantrowitz NE, Schmittger I, Schwarzkopf A, Fitzgerald PJ, Popp RL. Rapid, semiautomated technique for estimating left ventricular volume. AM HEART J 1983;106:521.

13. Folland ED, Parisi AF, Moynihan PF, Jones DR, Feldman CL, Tow DE. Assessment of left-ventricular ejection fraction and volumes by real-time, two-dimensional echocardiography. Circulation 1979;60:760.

14. Silverman NH, Ports TA, Snider AR, Schiller NB, Carlsson $E$, Heibron DC. Determination of left ventricular volume in children: Echocardiographic and angiographic comparisons. Circulation 1980;62:548.

15. Schiller NB, Acquatella H, Ports TA, Parmley WW, et al. Left ventricular volume from paired biplane two-dimensional echocardiography. Circulation 1979;60:547.

16. Mullins CB, Mason DT, Ashburn WL, Ross J. Determinaiton of ventricular volume by radioisotope-angiography. Am J Cardiol 1969;24:72.

17. Uren RF, Newman HN, Hutton BF, Cormack J, Berstein L, Leonard A, Dyer I. Geometric determination of left ventricular volume from gated blood-pool studies using a slant-hole collimator. Radiology 1983;147:541.

18. Massie BM, Kramer BL, Gertz EW, Henderson SG. Radionuclide measurement of left ventricular volume: Comparison of geometric and count-based methods. Circulation 1982;65: 725 .
19. Dehmer GJ, Lewis SE, Hillis LD, Twieg D, Falkoff M, Parkey RW, Willerson JT. Nongeometric determination of left ventricular volumes from equilibrium blood pool scans. Am J Cardiol 1980;45:293.

20. Links JM, Becker LC, Schindledecker JG, Guzman P, Burow RD, Nickoloff EL, Adderson PO, Wagner HN. Measurement of absolute left ventricular volume from gated blood pool studies. Circulation 1982;65:82.

21. Rabinovitch MA, Kalff V, Pitt B, Thrall JH, et al. Countbased left ventricular volume determination utilizing a left posterior oblique view for attenuation correction. Radiology 1984;150:813.

22. McNamara MT, Higgins CB, Schechtmann N, Botvinick E, Lipton M, Chatterjee K, Amparo EGG. Detection and characterization of acute myocardial infarction in man with use of gated magnetic resonance. Circulation 1985;71:717.

23. Gootman N, Rudolph AM, Buckley NM. Effects of angiographic contrast media on cardiac function. Am J Cardiol 1970;25:59.

24. Slinker BK, Glantz SA. The accuracy of inferring left ventricular volume from dimension depends on the frequency of information needed to answer a given question. Circ Res 1985;56:161.

25. Murphy WA, Gutierrez FR, Levitt RG, Glazer HS, Lee JKT. Oblique views of the heart by magnetic resonance imaging. Radiology 1985;154:225.

26. Feiglin DH, George CR, MacIntyre WJ, O'Donnell JK, Go RT, Paulicek W, Meaney TF. Gated cardiac magnetic resonance structural imaging: Optimization by electronic axial rotation. Radiology 1985;154:129.

27. Rzedzious R, Mansfield P, Doyle M, Guilfoyle D, Chapman B, Coupland RE, Chrispin A, Small P. Real-time nuclear magnetic resonance clinical imaging in paediatrics. Lancet 1983;2:1281.

28. Go RT, MacIntyre WJ, Yeung HN, Kramer DM, Geisenger M, Chilcote W, George C, O'Donnell IK, Moodie DS, Meaney TF. Volume and planar gated cardiac magnetic resonance imaging: A correlative study of normal anatomy with thallium-201 SPECT and cadaver sections. Radiology 1984; 150:129.

29. van Dijk IP, van der Menlen IP, Pettigrew RI, Blumm R, Dannels W, Doornbos J. Dynamic studies of cardiac motion and flow with a fast multiphase MRI technique (abstr). J Am Coll Cardiol 1986;7:197A. 